\title{
A Proposed Stochastic Finite Difference Approach Based on Homogenous Chaos Expansion
}

\author{
O. H. Galal \\ Engineering Mathematics and Physics Department, Faculty of Engineering, Fayoum University, Fayoum 63514, Egypt \\ Correspondence should be addressed to O. H. Galal; ohgalal2010@yahoo.com
}

Received 19 March 2013; Revised 24 June 2013; Accepted 26 June 2013

Academic Editor: Livija Cveticanin

Copyright (c) 2013 O. H. Galal. This is an open access article distributed under the Creative Commons Attribution License, which permits unrestricted use, distribution, and reproduction in any medium, provided the original work is properly cited.

\begin{abstract}
This paper proposes a stochastic finite difference approach, based on homogenous chaos expansion (SFDHC). The said approach can handle time dependent nonlinear as well as linear systems with deterministic or stochastic initial and boundary conditions. In this approach, included stochastic parameters are modeled as second-order stochastic processes and are expanded using KarhunenLoève expansion, while the response function is approximated using homogenous chaos expansion. Galerkin projection is used in converting the original stochastic partial differential equation (PDE) into a set of coupled deterministic partial differential equations and then solved using finite difference method. Two well-known equations were used for efficiency validation of the method proposed. First one being the linear diffusion equation with stochastic parameter and the second is the nonlinear Burger's equation with stochastic parameter and stochastic initial and boundary conditions. In both of these examples, the probability distribution function of the response manifested close conformity to the results obtained from Monte Carlo simulation with optimized computational cost.
\end{abstract}

\section{Introduction}

Stochastic processes are prevalent in nature. They affect all physical phenomena both from external and internal sources. Stochastic excitation and stochastic boundary conditions are a couple of examples of these external sources, while internal inherent random material heterogeneity also exists. To resolve these sources of stochastic inputs, stochastic differential equation is used for mathematical modeling. Methods of solution to these models are classified into two, statistical and nonstatistical. Monte Carlo simulation (MCS) is one of the most popular among the statistical methods of solution in use. As for the nonstatistical methodologies, these include stochastic perturbation method (SPM), stochastic finite element method (SFEM), and stochastic difference method (SFDM), the latter of which is the focus of this paper.

The nonstatistical methods' expansion process involves the discretization of input random fields. A projection scheme is further used resulting in deterministic equations solved by merely using one of the deterministic methods of solutions. Among these nonstatistical methods, SFEM is attracting the most attention. It has been applied to solve a wide range of problems especially in the field of structure mechanics (see, e.g., $[1,2]$ ). In contrast, FDM is rarely studied in the field of stochastic analysis. These rare studies include Kaminski [3] which introduced a second-order perturbation, second probabilistic moment analysis in the context of FDM. This technique uses the perturbation method to expand the input random fields and was applied to time dependent linear problems with a small range of variability, where the first two statistical moments of the response were calculated. In Kamiński's later works, he introduced a generalized version of this technique [4] which calculated higher probabilistic moments for any random dispersion of input variables. Ma et al. [5] presented a numerical method for a class of forward backward stochastic differential equations (FBSDEs). Their method is based on the four step scheme using a Hermitespectral method to approximate the solution to the decoupling quasilinear PDE on the whole space. Hovanessian and Chang [6] solved a second-order linear differential equation representing a series RLC network with step function, sinusoidal and stochastic inputs. Soheili et al. [7] later introduced two explicit finite difference schemes in order to approximate 
the solution of stochastic partial differential equations of Ito type.

This paper utilizes the homogenous chaos expansion in the context of finite difference method (SFDHC). In this technique, the random inputs are discretized using Karhunen-Loève (KL) expansion, while the response is represented in terms of homogenous chaos (HC) expansion then a Galerkin projection scheme is applied to generate a system of deterministic equations, which can then be solved using classical deterministic FDM. Enriching other popular methods like SFEM, the proposed technique can handle time dependent nonlinear as well as linear problems with stochastic or deterministic initial and boundary conditions using less computational cost. Moreover, results are obtained in the form of a probability distribution function (pdf) of the response at the grid points where the extreme values and statistical moments can be easily evaluated, an obvious improvement over the usual solution representation of merely mean values and standard deviations. Two illustrative problems are used to validate the efficiency of the proposed technique, the first one being linear diffusion equation with stochastic parameter and the second is the nonlinear Burger's equation with stochastic parameter and stochastic initial and boundary conditions. Both of these illustrative problem results are compared with MCS results. The comparison showed close conformity between SFDHC and MCS with optimized computational cost in favor of SFDHC.

\section{Random Field Discretization}

When one or more of the physical parameters are modelled as random fields, it is essential, first, to represent them by an enumerable set of random variables. Quite a number of random field discretization techniques were cited in the literature $[1,8]$. However, during the past two decades, KL and $\mathrm{HC}$ expansions have risen as two of the most practical techniques.

2.1. Karhunen-Loève Expansion. KL expansion is dependent on the covariance kernel [1]. Let $\alpha(x ; \theta)$ be a random process and $C_{\alpha \alpha}\left(x_{1}, x_{2}\right)$ be its correlation function. The discretized version of this field is written as

$$
\alpha(x ; \theta)=\bar{\alpha}(x)+\sum_{i=1}^{\infty} \sqrt{\lambda_{i}} f_{i}(x) \xi_{i}(\theta),
$$

where $\bar{\alpha}(x)$ denotes the mean value of $\alpha(x ; \theta),\left\{\xi_{i}(\theta)\right\}_{i=1}^{\infty}$ is a set of uncorrelated random variables, and $\lambda_{i}$ and $f_{i}(x)$ are the eigen values and the eigen functions, respectively. The said eigen value and eigen function are evaluated by solving Fredholm integral equation:

$$
\int_{D} C_{\alpha \alpha}\left(x_{1}, x_{2}\right) f_{i}\left(x_{1}\right) d x_{1}=\lambda_{i} f_{i}\left(x_{2}\right)
$$

where $D$ is the spatial domain over which $\alpha(x ; \theta)$ is defined, and $x_{1}, x_{2} \in D$. In the computational implementation of the expansion, (1) is truncated at the $M$ th term to reach the finitedimensional approximation:

$$
\alpha(x ; \theta)=\bar{\alpha}(x)+\sum_{i=1}^{M} \sqrt{\lambda_{i}} f_{i}(x) \xi_{i}(\theta) .
$$

2.2. Homogeneous Chaos Expansion. The solution process for stochastic differential equations is not known a priori, which means that its covariance function is unknown. KL expansion is, in this case, inapplicable requiring $\mathrm{HC}$ expansion instead. HC expansion considers a probability space $(\Omega, \kappa, P)$, where $\Omega$ denotes the space of basic outcomes, $\kappa$ is the $\sigma$-algebra associated with $\Omega$, and $P$ is a probability measure on $\kappa$. Considering some physical domain $D \subset R^{d} \times T,(d=$ $1,2$, or 3$)$, this can be a combination of spatial and temporal dimensions. A stochastic process (SP) can be seen as a scalar or vector-valued function $u(x, t, \theta): D \times \Omega \rightarrow R^{b}$, where $x$ is an element of the physical space, $t$ denotes the time, and $\theta$ is a point in the sample space $\Omega$. Because of the infinite-dimensional nature of the probability space, it has to be discretized by using a finite number of random variables $\left\{\xi_{i}(\theta)\right\}_{i=1}^{n}, n \in N$.

$\mathrm{HC}$ expansion polynomials are functions in the infinite set of random variables $\left\{\xi_{i}(\theta)\right\}_{i=1}^{i=\infty}$ and therefore of infinite dimensions. A finite subset $\left\{\xi_{\lambda_{i}}(\theta)\right\}_{i=1}^{i=n}$ was chosen to build these polynomials; hence, the finite-dimensional polynomials of any order $p$ will be a subset of the infinite-dimensional polynomials of the same order. The convergence of the finitedimensional polynomials is related to $n$ as well as to the choice of the subset $\left\{\xi_{\lambda_{i}}(\theta)\right\}_{i=1}^{i=n}$; the choice of this subset can be taken according to K-L expansion of the random process to ensure the convergence of a polynomial chaos (PC) expansion in the mean-square sense. As shown in $[1,9,10]$, the solution process can be expressed as a summation of nonlinear functionals of the set $\left\{\xi_{i}(\theta)\right\}_{i=1}^{\infty}$ multiplied by deterministic constants. These functionals can be expanded as a set of polynomials of second-order Gaussian random variables, $\xi_{i}(\theta) \in N(0,1)$, referred to as polynomial chaos. Hence, the random process, which represents the random field, can be expressed as

$$
\begin{aligned}
u(\theta)= & a_{0} \Gamma_{0}+\sum_{i_{1}=1}^{\infty} a_{i_{1}} \Gamma_{1}\left(\xi_{i_{1}}(\theta)\right) \\
& +\sum_{i_{1}=1}^{\infty} \sum_{i_{2}=1}^{\infty} a_{i_{1} i_{2}} \Gamma_{2}\left(\xi_{i_{1}}(\theta), \xi_{i_{2}}(\theta)\right)+\cdots ;
\end{aligned}
$$

in which $\Gamma_{n}\left(\xi_{i_{1}}(\theta), \ldots, \xi_{i_{n}}(\theta)\right)$ is the polynomial chaos of order $n$ in the set of variables $\left(\xi_{i_{1}}(\theta), \ldots, \xi_{i_{n}}(\theta)\right)$ given in [10]. Truncating at the $P$ th order, (4) can be then written as

$$
u\left(x, t, \xi_{M}(\theta)\right)=\sum_{i=0}^{P} \bar{u}_{i}(x, t) \psi_{i}\left[\left\{\xi_{M}(\theta)\right\}\right]
$$

or simply

$$
u(x, t, \xi)=\sum_{i=0}^{P} \bar{u}_{i} \psi_{i}
$$


The total number of these polynomials is $(P+1)$, where

$$
P+1=\frac{(M+p) !}{p !(M) !} .
$$

In (5), $\bar{u}_{i}(x, t)$ is a set of deterministic coefficients dependent on both $x$ and $t, \psi_{i}\left[\left\{\xi_{M}\right\}\right]$ is a set polynomials (up to order $p$ ) in the set of random variables $\left(\xi_{i_{1}}(\theta), \ldots, \xi_{i_{M}}(\theta)\right)$, and $M$ refers to the number of terms in KL expansion. For example, if $M=p=2$, the set of second-order-seconddegree PC consists of 6 elements; $\left\{1, \xi_{1}, \xi_{2}, \xi_{1}^{2}-1, \xi_{1} \xi_{2}, \xi_{2}^{2}-\right.$ 1\}. Also, if $M=4, p=2$, the set of second-orderfourth-degree PC consists of 15 elements; $\left\{1, \xi_{1}, \xi_{2}, \xi_{3}, \xi_{4}, \xi_{1}^{2}-\right.$ $\left.1, \xi_{1} \xi_{2}, \xi_{1} \xi_{3}, \xi_{1} \xi_{4}, \xi_{2}^{2}-1, \xi_{2} \xi_{3}, \xi_{2} \xi_{4}, \xi_{3}^{2}-1, \xi_{3} \xi_{4}, \xi_{4}^{2}-1\right\}$. Also, $\psi_{i}\left[\left\{\xi_{n}\right\}\right]$ have the following orthogonality properties:

$$
\begin{gathered}
\left\langle\psi_{i}\left[\left\{\xi_{n}\right\}\right]\right\rangle=1 \quad \text { for } i=0 \text { and zero otherwise, } \\
\left\langle\psi_{i}\left[\left\{\xi_{n}\right\}\right] \psi_{j}\left[\left\{\xi_{n}\right\}\right]\right\rangle=\delta_{i j}\left\langle\psi_{i}^{2}\left[\left\{\xi_{n}\right\}\right]\right\rangle .
\end{gathered}
$$

According to the Cameron-Martin theorem [11], for a fixed value of $x$ and $t$, this expansion converges to any $L_{2}(\Omega)$ functional in the $L_{2}(\Omega)$ sense. This implies that the application of polynomial chaos is restricted to those stochastic processes with finite second-order moments (finite variance), and this applies to most physical processes yielding

$$
\int_{\theta \in \Omega}|u(x, t, \theta)|^{2} d P(\theta)<\infty
$$

\section{A Proposed Stochastic Finite Difference Approach Based on Homogenous Chaos Expansion (SDFHC)}

In this proposed technique, the involved random parameters in SDE are expanded using KL expansion, which is truncated at the $M$ th term. The response function $u$ is, in turn, expanded using $\mathrm{HC}$ expansion since its covariance kernel is not known a priori. Applying Galerkin projection scheme through multiplying both sides of the resulting equation by $\psi_{j}$ and taking the statistical average yields a deterministic system of equations in $\bar{u}_{i}(x, t)$ which can be solved simply using the classical deterministic FDM. Consider

$$
\Lambda(x, t, \xi(\theta) ; u)=f(x, t, \xi(\theta))
$$

where $f(x, t, \xi(\theta))$ is a random external excitation and $\Lambda$ is a differential operator involving differentiation in space and/or time which can be nonlinear and defined on the product space $D \times \Omega$. Also, $u$ satisfies the initial and boundary conditions, deterministic or stochastic, and they are defined on some section of the boundary $\partial D_{1} \in \partial D$. Using (6) to expand $u$ in (10) yields

$$
\Lambda\left(x, t, \xi(\theta) ; \sum_{i=0}^{P} \bar{u}_{i} \psi_{i}\right)=f(x, t, \xi(\theta)) .
$$

Multiply the different orthogonal polynomials of the finite expansion in (11) and get statistical average results to

$$
\begin{array}{r}
\left\langle\Lambda\left(x, t, \xi ; \sum_{i=0}^{P} \bar{u}_{i} \psi_{i}\right), \psi_{j}\right\rangle=\left\langle f(x, t, \xi), \psi_{j}\right\rangle, \\
j=0,1,2, \ldots, P .
\end{array}
$$

From (12), it should be noted that $(P+1)$ set of coupled equations $\bar{u}_{i}(x, t)$ is generated. This most recent step is actually Galerkin projection in random space. The projection ensures the orthogonality of the residual to the functional space spanned by the finite-dimensional basis $\left\{\psi_{i}\right\}_{i=0}^{i=P}$. As predicted, a strictly deterministic $\bar{u}_{i}(x, t)$ was achieved which would merely require a classical FDM-based solution in space and time. The resulting feature is mainly due to the averaging inherent in the projection, eliminating the randomness of the system model. It could also be noted that no additional errors, except for truncation errors, manifest from this step.

For a numerical solution using FDM, the physical domain $D$ is covered by a grid of mesh points $(x, t)=(m \Delta x, n \Delta t)$ where $\Delta x$ and $\Delta t$ are mesh parameters that are relatively infinitesimal and $m$ and $n$ are positive integers. The approximate solution of $\bar{u}_{i}(x, t)$ at these mesh points will be denoted by $\bar{u}_{i}^{(m, n)}$; provided that the derivatives of $\bar{u}_{i}(x, t)$ have been replaced by finite difference quotients. Moreover, the choice of both $\Delta x$ and $\Delta t$ must satisfy all necessary conditions of the deterministic FD scheme like convergence, stability, and consistency. Once $\bar{u}_{i}(x, t)$ is obtained at every point in the mesh, (6) can be used to get the solution vector where the mean is given as

$$
\langle u(x, t, \xi)\rangle=\bar{u}_{0}(x, t),
$$

and the covariance matrix for two points $u$ and $v$ is

$$
\operatorname{Cov}(u, v)=\left\langle(u-\langle u\rangle)(v-\langle v\rangle)^{T}\right\rangle,
$$

and the resulting variance vector of each point is

$$
\operatorname{Var}(u(x, t, \xi))=\sum_{i=1}^{P}\left\langle\psi_{i}^{2}\right\rangle \bar{u}_{i}^{2}(x, t) .
$$

\section{Illustrative Example I: The Stochastic Diffusion Equation}

In order to validate the efficiency of the proposed SFDHC technique, stochastic diffusion equation is evaluated under the effect of random parameter $\gamma(x, \theta)$. The results are then compared with that obtained from MCS method.

4.1. Problem Formulation. Consider the diffusion equation [12] with random parameter $\gamma(x, \theta)$ in the form

$$
\frac{\partial u(x, t, \xi)}{\partial t}=\gamma(x, \theta) \frac{\partial^{2} u(x, t, \xi)}{\partial x^{2}},
$$


where: $x \in[0, L]$ and $t \geq 0$. Also, consider the case of deterministic initial and boundary conditions

$$
u(0, t)=q_{1}(t), \quad u(L, t)=q_{2}(t), \quad u(x, 0)=h(x) .
$$

Moreover, the random parameter can be written as:

$$
\gamma(x, \theta)=\gamma(1+\alpha(x ; \theta))
$$

where $\alpha(x ; \theta)$ is SP which expresses the spatial randomness in the parameter $\gamma(x, \theta)$. Expanding both $\alpha(x ; \theta)$ and $u(x, t, \xi)$ using (3) and (6), respectively, yields

$$
\sum_{i=0}^{P} \frac{\partial \bar{u}_{i}}{\partial t} \psi_{i}=\gamma\left(1+\sum_{i=1}^{M} \sqrt{\lambda_{j}} f_{j}(x) \xi_{j}\right) \sum_{i=0}^{P} \frac{\partial^{2} \bar{u}_{i}}{\partial x^{2}} \psi_{i}
$$

Using Galerkin projection scheme and multiplying both sides of (18) by $\psi_{j}$ then taking the statistical average yields

$$
\begin{aligned}
& \sum_{i=0}^{P} \sum_{j=0}^{P} b_{i j} \frac{\partial \bar{u}_{j}}{\partial t} \\
& \quad=\gamma \sum_{i=0}^{P} \sum_{j=0}^{P} b_{i j} \frac{\partial^{2} \bar{u}_{j}}{\partial x^{2}}+\gamma \sum_{i=0}^{P} \sum_{j=0}^{P} \sum_{k=1}^{M} d_{i j k} \sqrt{\lambda_{k}} f_{k}(x) \frac{\partial^{2} \bar{u}_{j}}{\partial x^{2}}
\end{aligned}
$$

where

$$
b_{i j}=\left\langle\psi_{i} \psi_{j}\right\rangle, \quad d_{i j k}=\left\langle\psi_{i} \psi_{j} \xi_{k}\right\rangle .
$$

Replacing the derivatives of $\bar{u}_{i}$ by their difference quotients at the mesh points leads to

$$
\begin{aligned}
& \frac{1}{\Delta t} \sum_{i=0}^{P} \sum_{j=0}^{P} b_{i j}\left(\bar{u}_{j}^{(m, n+1)}-\bar{u}_{j}^{(m, n)}\right) \\
& =\frac{\gamma}{(\Delta x)^{2}} \sum_{i=0}^{P} \sum_{j=0}^{P} b_{i j}\left(\bar{u}_{j}^{(m+1, n)}-2 \bar{u}_{j}^{(m, n)}+\bar{u}_{j}^{(m-1, n)}\right) \\
& \quad-\frac{\gamma}{(\Delta x)^{2}} \sum_{i=0}^{P} \sum_{j=0}^{P} \sum_{k=1}^{M} d_{i j k} \sqrt{\lambda_{k}} f_{k}(x) \\
& \quad \times\left(\bar{u}_{j}^{(m+1, n)}-2 \bar{u}_{j}^{(m, n)}+\bar{u}_{j}^{(m-1, n)}\right) .
\end{aligned}
$$

Introducing $\mu=\gamma \Delta t(\Delta x)^{-2}$ and solving the last equation for $\bar{u}_{i}^{(m, n+1)}$ yields

$$
\begin{aligned}
& \sum_{i=0}^{P} \sum_{j=0}^{P} b_{i j} \bar{u}_{j}^{(m, n+1)} \\
& =\sum_{i=0}^{P} \sum_{j=0}^{P} b_{i j} \bar{u}_{j}^{(m, n)} \\
& \quad+\gamma \mu \sum_{i=0}^{P} \sum_{j=0}^{P} b_{i j}\left(\bar{u}_{j}^{(m+1, n)}-2 \bar{u}_{j}^{(m, n)}+\bar{u}_{j}^{(m-1, n)}\right) \\
& \quad+\gamma \mu \sum_{i=0}^{P} \sum_{j=0}^{P} \sum_{k=1}^{M} d_{i j k} \sqrt{\lambda_{k}} f_{k}(x) \\
& \times\left(\bar{u}_{j}^{(m+1, n)}-2 \bar{u}_{j}^{(m, n)}+\bar{u}_{j}^{(m-1, n)}\right),
\end{aligned}
$$

which can be solved using an explicit scheme to find $\bar{u}_{i}(x, t)$ at the different mesh points.

4.2. Numerical Implementation and Results. To implement the results obtained in (22), consider $\alpha(x ; \theta)$ as a secondorder Gaussian SP with exponential covariance kernel given in [1]

$$
\operatorname{Cov}\left(x_{1}, x_{2}\right)=\sigma^{2} \exp \left(-\frac{\left|x_{1}-x_{2}\right|}{l_{x}}\right)
$$

where $\sigma$ denotes the coefficient of variation (COV) of the random field (which is constant) and $l_{x}$ is the correlation length. Assume $\sigma^{2}=0.01$ and both $l_{x}$ and $\gamma$ are unity. Also, let the initial and boundary conditions be considered as

$$
q_{1}(t)=q_{2}(t)=0, \quad h(x)=\sin \frac{\pi x}{L},
$$

where $L=1, x \in[0,1]$, and $t \in[0,0.04]$. Consider also the mesh parameters $\Delta x=0.1$ and $\Delta t=0.004$ so that $\mu \leq 1 / 2$, which is a necessary condition for the convergence of the FD scheme. This, produces a moderately course mesh of 11 joints in both $x$ and $t$ directions, with a total number of unknown joints NP $=90$ (without the boundary ones). The problem is solved using MCS [13], where $\alpha(x ; \theta)$ is expanded using $\mathrm{KL}$ expansion with $M=6$. A large sample size of $1 \times 10^{6}$ is then used to generate the random variables where exact deterministic solution, given in [12], is evaluated for each input. This in turn, becomes a benchmark for the proposed technique. HC of second and fourth degrees, SFD2 and SFD4, respectively, with order two were employed to derive the SFDHC's solution. The same sample size was then used to generate the HC polynomials. The pdf of the solution process $u$ is plotted using MCS on SFD2 and SFD4 for 2 arbitrary points, point $A$ on the midspan at $(0.5,0.024)$ and $B$ near the boundary at $(0.9,0.036)$, as shown in Figures 1 and 2, respectively. The mean and standard deviation (SD) of $u$ are also plotted at an arbitrary time level $(t=0.04)$ as shown in Figures 3 and 4 . 


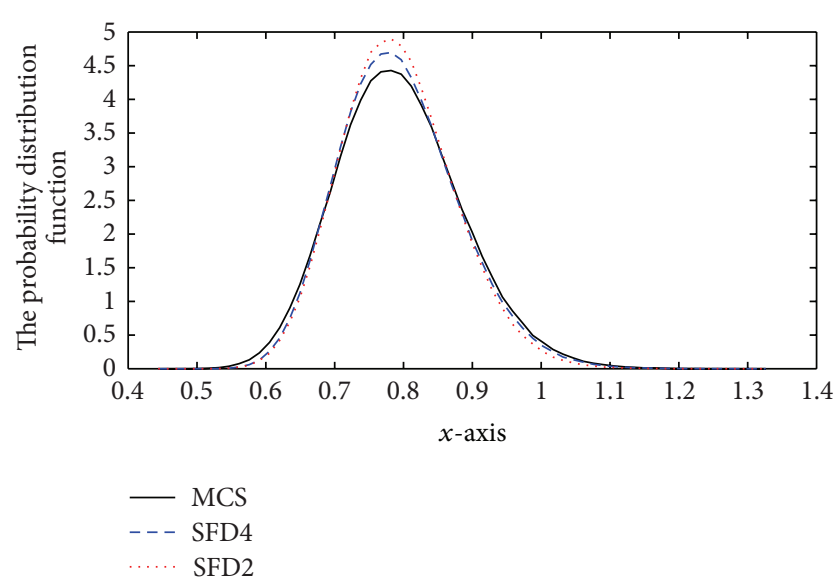

Figure 1: The pdf at point $A$ using SFDHC and MCS.

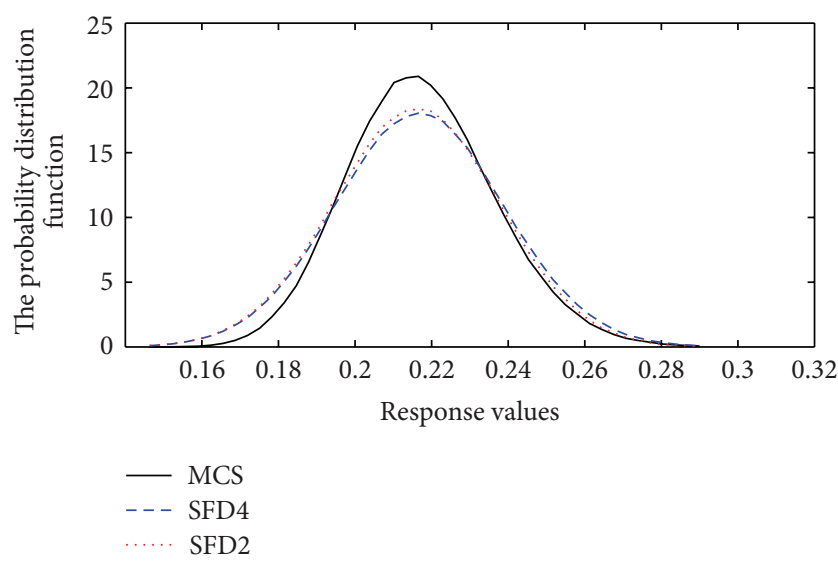

FIgUre 2: The pdf at point $B$ using SFDHC and MCS.

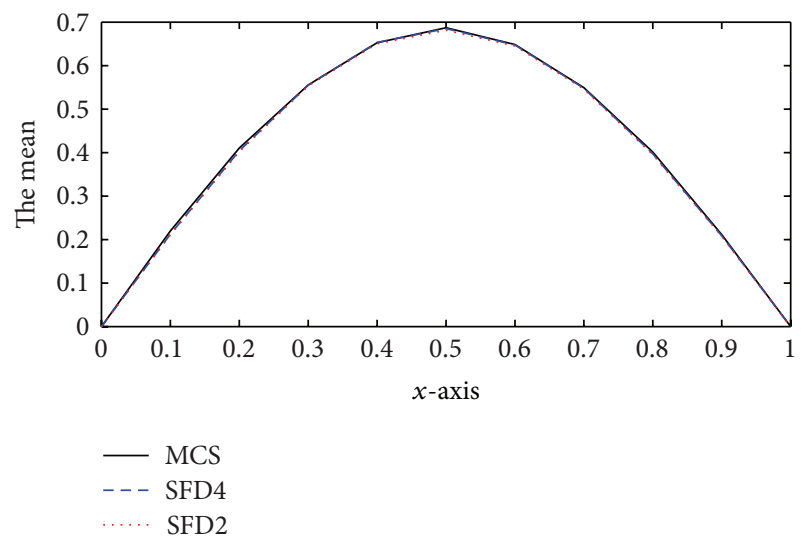

Figure 3: The mean at $t=0.04$ using SFDHC and MCS.

4.3. Error Analysis. The resulting response from the SFDHC technique, with two degrees of the polynomial chaos (SFD2 and SFD4), and MCS results (incorporating the exact solution given in [12]) were compared in terms of the maximum and minimum errors, their locations, and average error. From this, the relative accuracy of SFDHC technique is then recorded as shown in Table 1.

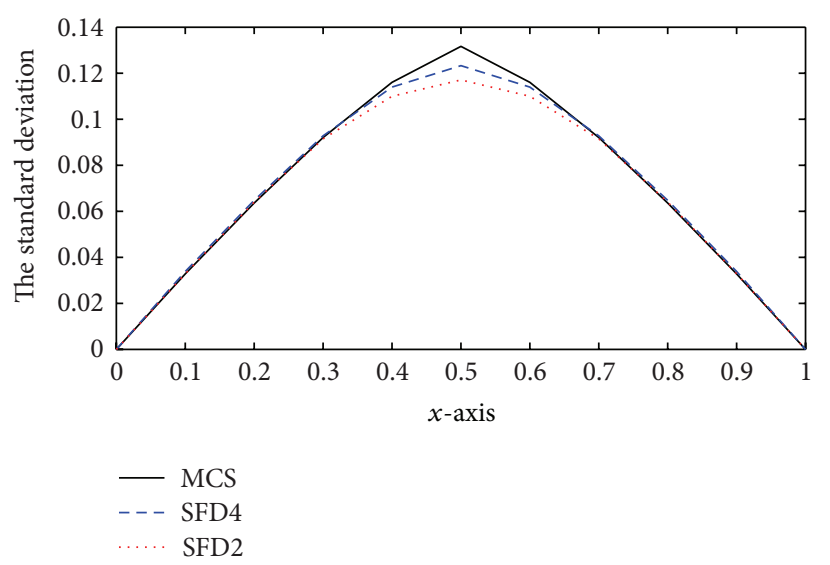

FIgURE 4: The SD at $t=0.04$ using SFDHC and MCS.

Note that the average error for the mean value and SD was calculated through the summation of the errors over all points and then dividing the sum by the number of points NP. Table 1 shows good convergence of the SFDHC technique with increasing the degree of the used polynomials. Evidently, for SFD2, the mean value and SD errors were maximum at the midspan, while they were minimum near the boundary. For SFD4, however, the mean value error has the same positions as SFD2, while the SD error is minimum around the first and third quarters. This conclusion is made from this particular problem with their corresponding inputs. Generally, from the insignificant errors recorded, the SFDHC technique exhibits very high accuracy for calculating the mean value and SD.

\section{Illustrative Example II: Stochastic Nonlinear Burger's Equation}

To illustrate the proposed SFDHC technique on a nonlinear case, consider stochastic nonlinear Burger's equation (SBE) [14]. In addition, let us involve random parameters and expose them to deterministic or stochastic random excitation with deterministic or stochastic boundary and initial conditions.

5.1. Problem Formulation. With a random parameter $\gamma(x, \theta)$ and random excitation $p(x, t, \theta)$, the nonlinear stochastic Burger's equation can then be written as

$$
\begin{aligned}
& \frac{\partial u(x, t, \xi)}{\partial t}+u(x, t, \xi) \frac{\partial u(x, t, \xi)}{\partial x}-\gamma(x, \theta) \frac{\partial^{2} u(x, t, \xi)}{\partial x^{2}} \\
& \quad=p(x, t, \theta) .
\end{aligned}
$$

Given that the domain and initial and boundary conditions are consistent with Section 4.2 and the excitation functions can be written as

$$
p(x, t, \theta)=p(x, t)(1+\beta(x ; \theta)),
$$

where $\beta(x ; \theta)$ is an SP expressing the spatial randomness in the excitation function. Also, $\beta(x ; \theta)$ can be expanded 
TABLE 1: Errors and accuracy for the stochastic diffusion equation.

\begin{tabular}{|c|c|c|c|c|c|c|}
\hline & \multicolumn{3}{|c|}{ The mean } & \multicolumn{3}{|c|}{ The standard deviation } \\
\hline & $\begin{array}{c}\text { Min. } \\
\text { error/location }\end{array}$ & $\begin{array}{c}\text { Max. } \\
\text { error/location }\end{array}$ & $\begin{array}{c}\text { Average } \\
\text { error/accuracy }\end{array}$ & $\begin{array}{c}\text { Min. } \\
\text { error/location }\end{array}$ & $\begin{array}{c}\text { Max. } \\
\text { error/location }\end{array}$ & $\begin{array}{c}\text { Average } \\
\text { error/accuracy }\end{array}$ \\
\hline \multirow[b]{2}{*}{ SFD2 } & $0.059 \%$ & $0.696 \%$ & $0.317 \%$ & $0.1412 \%$ & $11.09 \%$ & $2.841 \%$ \\
\hline & $\begin{array}{c}(0.1,0.004) \\
(0.9,0.004)\end{array}$ & $(0.5,0.04)$ & $99.683 \%$ & $\begin{array}{l}(0.1,0.04) \\
(0.9,0.04)\end{array}$ & $(0.5,0.04)$ & $97.159 \%$ \\
\hline \multirow[b]{2}{*}{ SFD4 } & $0.0035 \%$ & $0.221 \%$ & $0.128 \%$ & $0.0024 \%$ & $6.3645 \%$ & $1.393 \%$ \\
\hline & $\begin{array}{c}(0.1,0.004), \\
(0.9,0.004)\end{array}$ & $(0.5,0.04)$ & $99.872 \%$ & $\begin{array}{c}(0.3,0.024), \\
(0.7,0.024)\end{array}$ & $(0.5,0.04)$ & $98.607 \%$ \\
\hline
\end{tabular}

using (3) with $\gamma_{j}$ and $g_{j}(x)$ as the eigen values and the eigen functions, associated with its covariance kernel, respectively. Expanding $u(x, t, \xi)$ as in (6) yields

$$
\begin{gathered}
\sum_{i=0}^{P} \frac{\partial \bar{u}_{i}}{\partial t} \psi_{i}+\sum_{i=0}^{P} \bar{u}_{i} \psi_{i} \sum_{j=0}^{P} \frac{\partial \bar{u}_{j}}{\partial x} \psi_{j}-\gamma\left(1+\sum_{i=1}^{M} \sqrt{\lambda_{j}} f_{j}(x) \xi_{j}\right) \\
\times \sum_{i=0}^{P} \frac{\partial^{2} \bar{u}_{i}}{\partial x^{2}} \psi_{i}=p(x, t)\left(1+\sum_{i=1}^{M} \sqrt{\gamma_{i}} g_{i}(x) \xi_{i}\right) .
\end{gathered}
$$

Multiplying both sides of (17) by $\psi_{k}$ and taking the statistical average yields

$$
\begin{aligned}
& \sum_{i=0}^{P} \sum_{j=0}^{P} b_{i j} \frac{\partial \bar{u}_{j}}{\partial t}+\sum_{i=0}^{P} \sum_{j=0}^{P} \sum_{k=0}^{P} c_{i j k} \bar{u}_{j} \frac{\partial \bar{u}_{k}}{\partial x} \\
& \quad-\gamma \sum_{i=0}^{P} \sum_{j=0}^{P} \sum_{k=1}^{M} d_{i j k} \sqrt{\lambda_{k}} f_{k}(x) \frac{\partial^{2} \bar{u}_{j}}{\partial x^{2}}-\gamma \sum_{i=0}^{P} \sum_{j=0}^{P} b_{i j} \frac{\partial^{2} \bar{u}_{j}}{\partial x^{2}} \\
& =p(x, t)+p(x, t) \sum_{i=0}^{P} \sum_{j=1}^{M} e_{i j} \sqrt{\gamma_{j}} g_{j}(x)
\end{aligned}
$$

where

$$
c_{i j k}=\left\langle\psi_{i} \psi_{j} \psi_{k}\right\rangle, \quad e_{i j}=\left\langle\psi_{i} \xi_{j}\right\rangle
$$

Replacing the derivatives of $\bar{u}_{i}$ by their difference quotients and solving the last equation for $\bar{u}_{i}^{(m, n+1)}$ yields

$$
\begin{aligned}
\sum_{i=0}^{P} \sum_{j=0}^{P} b_{i j} \bar{u}_{j}^{(m, n+1)} & \\
= & \sum_{i=0}^{P} \sum_{j=0}^{P} b_{i j} \bar{u}_{j}^{(m, n)} \\
& \quad-\frac{1}{2} \Delta x \mu \sum_{i=0}^{P} \sum_{j=0}^{P} \sum_{k=0}^{P} c_{i j k} \bar{u}_{j}^{(m, n)}\left(\bar{u}_{k}^{(m+1, n)}-\bar{u}_{k}^{(m-1, n)}\right)
\end{aligned}
$$

$$
\begin{aligned}
& +\gamma \mu \sum_{i=0}^{P} \sum_{j=0}^{P} \sum_{k=1}^{M} d_{i j k} \sqrt{\lambda_{k}} f_{k}(x) \\
& \times\left(\bar{u}_{j}^{(m+1, n)}-2 \bar{u}_{j}^{(m, n)}+\bar{u}_{j}^{(m-1, n)}\right) \\
& +\gamma \mu \sum_{i=0}^{P} \sum_{j=0}^{P} b_{i j}\left(\bar{u}_{j}^{(m+1, n)}-2 \bar{u}_{j}^{(m, n)}+\bar{u}_{j}^{(m-1, n)}\right) \\
& +\Delta t p(x, t)+\Delta t p(x, t) \sum_{i=0}^{P} \sum_{i=j}^{M} e_{i j} \sqrt{\gamma_{j}} g_{j}(x)
\end{aligned}
$$

5.2. Numerical Implementation and Results. The proposed technique will be applied to SBE in two cases: Case 1 using random parameter and, Case 2 using random initial and boundary conditions. Both Cases 1 and 2 are treated as follows.

Case 1. The same data used in Section 4.2 concerning the domain, the initial and boundary conditions, the mesh parameters, and $\alpha(x ; \theta)$ will be employed here. First, assume the $\mathrm{COV}$ as unity for error analysis and for Figures 5 and 6 . Then, some practical values $(0.05,0.10$, and 0.20$)$ will be considered in Figures 7 and 8. Both of SFD2 and SFD4 are used to solve (30), where the following results are obtained for points $C(0.5,0.036)$ and $D(0.9,0.036)$.

5.3. Error Analysis. As in Section 4.3, errors and accuracy are recorded as shown in Table 2.

Although the errors are slightly bigger than the linear case, the SFDHC technique still exhibits high performance for nonlinear problems.

Case 2. The proposed SFDHC technique can handle stochastic initial and boundary conditions expressed as

$$
u(x, t, \xi)=\sum_{i=0}^{P_{1}} \bar{u}_{i}(x, t) \chi_{i}(\xi)
$$

defined on some section of the boundary $\partial D_{1} \in \partial D$, where $P_{1} \leq P$ and both degree and order of $\chi_{i}$ are less than or equal 
TABLE 2: Errors and accuracy for the stochastic Burger's equation.

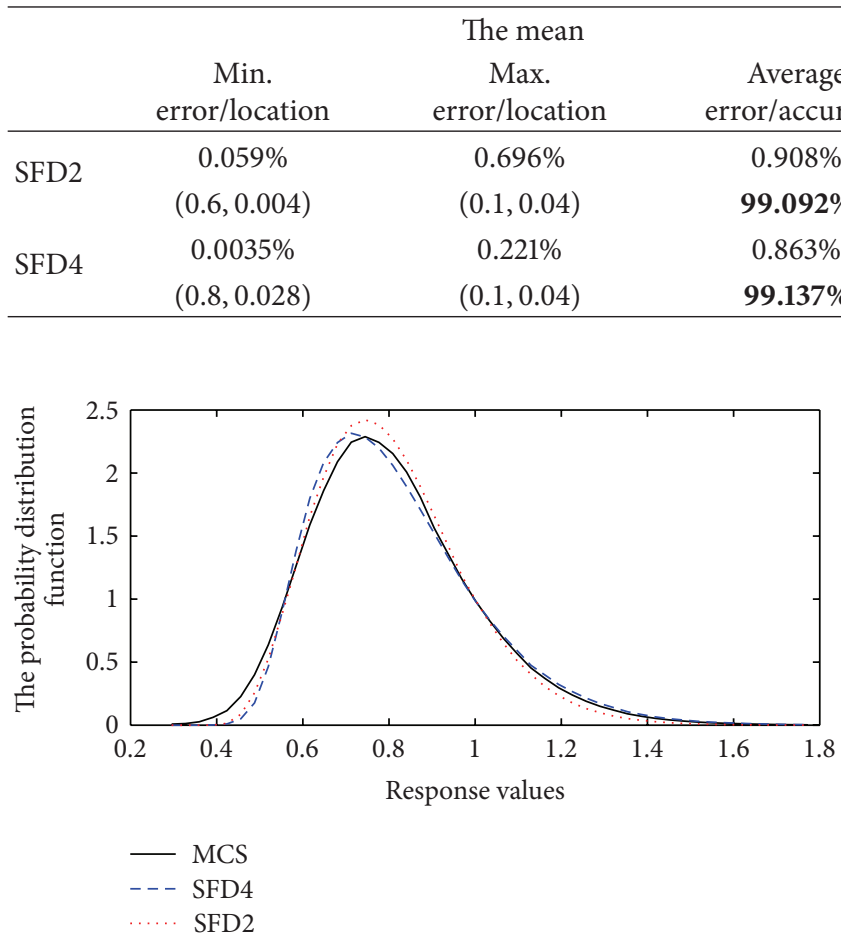

FIgure 5: The pdf at point $C$ using SFDHC and MCS.

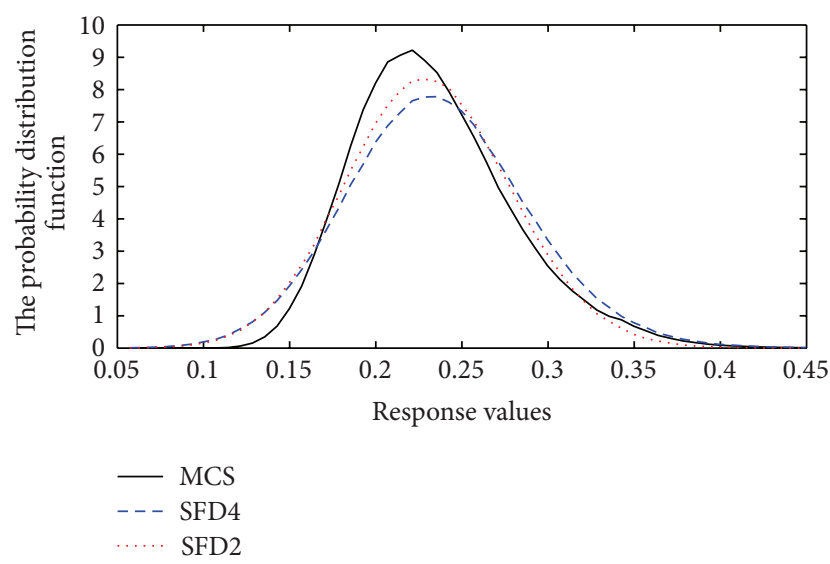

Figure 6: The pdf at point $D$ using SFDHC and MCS.

to those used for $\psi_{i}$. After executing the same procedure as in Case 1, it yields

$$
\begin{aligned}
\sum_{i=0}^{P} \sum_{j=0}^{P} b_{i j} \bar{u}_{j}^{(m, n+1)} & \\
= & \sum_{i=0}^{P} \sum_{j=0}^{P} b_{i j} \bar{u}_{j}^{(m, n)} \\
& -\frac{1}{2} \Delta x \mu \sum_{i=0}^{P} \sum_{j=0}^{P} \sum_{k=0}^{P} c_{i j k} \bar{u}_{j}^{(m, n)}\left(\bar{u}_{k}^{(m+1, n)}-\bar{u}_{k}^{(m-1, n)}\right)
\end{aligned}
$$

The standard deviation

Min. Max. Average error/location error/location error/accuracy

$0.1412 \% \quad 11.09 \% \quad 4.632 \%$

$(0.9,0.04) \quad(0.9,0.004) \quad \mathbf{9 5 . 3 6 8 \%}$

$\begin{array}{lll}0.0024 \% & 6.3645 \% & 2.973 \%\end{array}$

$\begin{array}{lll}(0.9,0.04) & (0.4,0.04) & \mathbf{9 7 . 0 2 7} \%\end{array}$

As a practical example, assume an initial condition with the form

$$
u(x, 0, \xi)=x(L-x)(1+\alpha(x ; \theta))
$$

and boundary conditions

$$
u(0, t, \xi)=\alpha(0 ; \theta), \quad u(L, t, \xi)=\alpha(L ; \theta)
$$

where $\alpha(x ; \theta)$ is the same Gaussian SP used in Section 4.2 with $\mathrm{COV}=1$. Then, $\bar{u}_{0}(x, 0)=x(L-x), \bar{u}_{i}(x, 0)=\sigma \sqrt{\lambda_{i}} f_{i}(x)$ for $1 \leq i \leq M$, and $\bar{u}_{i}(x, 0)=0$ for $i>M$. Also, $\bar{u}_{0}(0, t)=$ $\bar{u}_{0}(L, t)=0, \bar{u}_{i}(0, t)=\sigma \sqrt{\lambda_{i}} f_{i}(0), \bar{u}_{i}(L, t)=\sigma \sqrt{\lambda_{i}} f_{i}(L)$ for $1 \leq i \leq M$ and $\bar{u}_{i}(0, t)=\bar{u}_{i}(L, t)=0$ for $i>M$. Both of SFD4 and SFD2 are used to solve (32) with initial and boundary conditions given by (33) and (34), respectively. An MCS solution was provided through expanding the included stochastic processes using KL expansion then a sample of size 50,000 random numbers is used to generate the initial and boundary random values. Consequently, the problem is solved using FD forward scheme leading to 50,000 values of the stochastic response at each of the mesh points. A solution pdf for SFDHC is generated and then plotted for points $C(0.5,0.036)$ and $D(0.9,0.036)$ shown in Figures 9 and 10 .

Comparing with MCS, SFD4 shows better approximation for the response (as expected) since it uses 4 random variables in its expansion, while SFD2 uses only 2.

\section{Discussion and Conclusions}

As evidenced in this work, the SFDHC technique was designed to solve a wide range of time dependent nonlinear as well as linear problems with stochastic or deterministic initial and boundary conditions and exposed to deterministic or stochastic excitation. This technique uses HC expansion in compliment with FDM, providing the capacity to present the analysis result as pdf and not just the statistical moments of the response. Using two illustrative examples, as representative of linear and nonlinear problems, the proposed technique has proven to be highly efficient and 


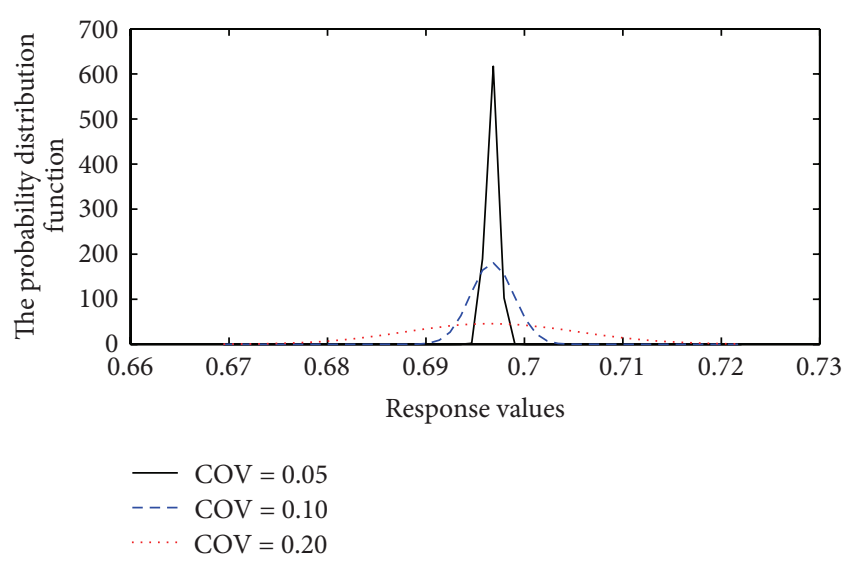

Figure 7: The pdf at point $C$ for different values of COV.

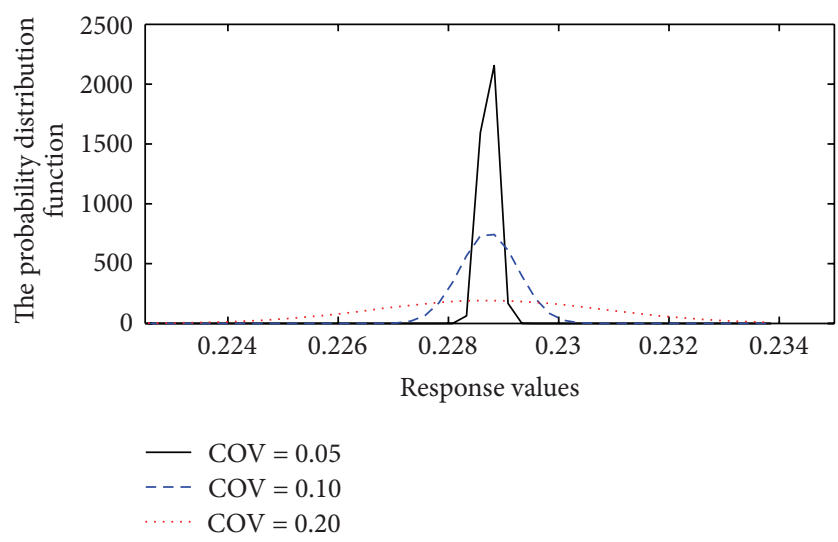

FIgURE 8: The pdf at point $D$ for different values of COV.

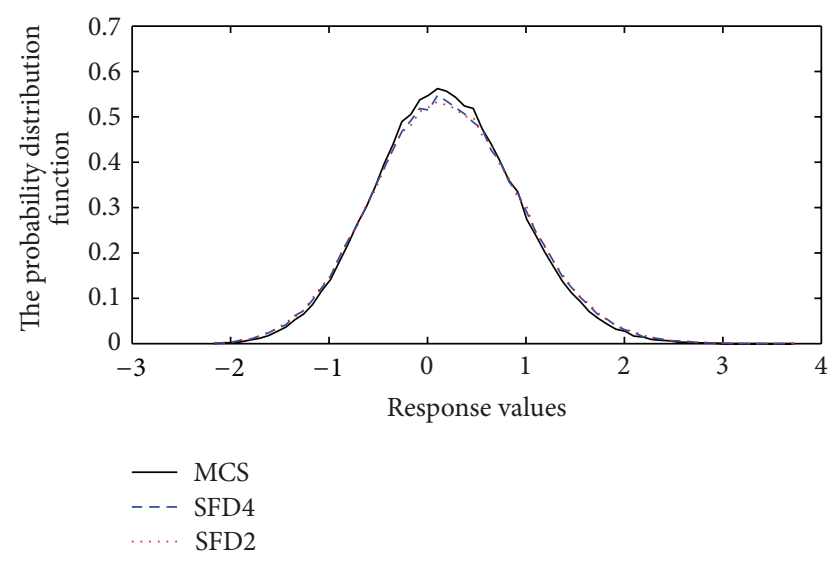

Figure 9: The pdf at point $C$ using SFD4 and SFD2.

computationally optimized. Using SFD4, the efficiency of this technique exceeds $98.6 \%$ for both mean and SD evaluations in the linear problem and exceeds $97 \%$ for the nonlinear one. Moreover, the computation times spent for SFDHC, on a Core-i3 computer, were 3.08 and 3.91 seconds for linear and nonlinear problems, respectively, as against 40.19 and 989.82 seconds for MCS, respectively, which means that $7.66 \%$ and $0.395 \%$ only of the execution time are in favor of SFDHC.

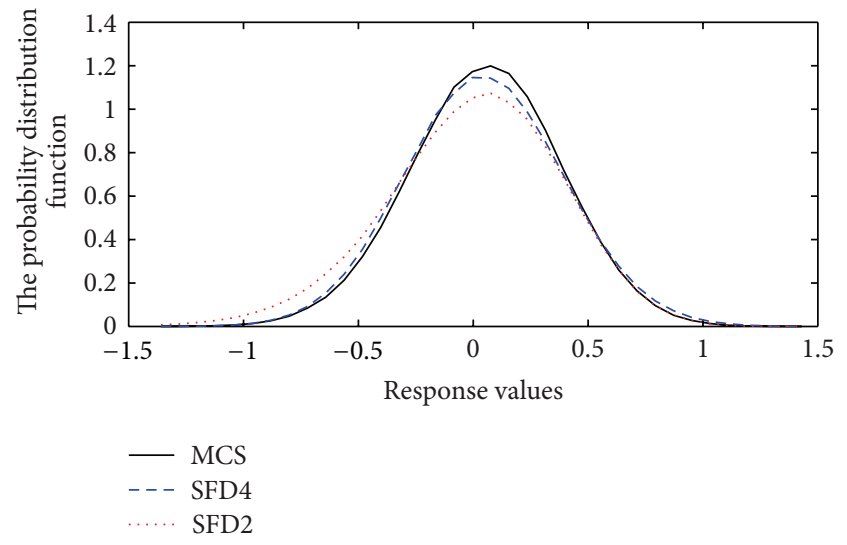

Figure 10: The pdf at point $D$ using SFDHC and MCS.

The overwhelming advantages manifested by the proposed technique open the door for the versatility manifested by FDM in the deterministic field into the stochastic field. FDM had shown its versatility in the deterministic field by directly dealing with time dependent and nonlinear systems through explicit or implicit schemes. These capabilities of FDM will be available in the stochastic field by the way of this proposed technique. The SFDHC technique can handle more complicated nonlinear problems in 2 and 3 dimensions with explicit or implicit schemes which are available in the FDM literature. However, the illustrative examples in this paper were onedimensional problems and were solved explicitly. Further research concerning the range of variability of random inputs, the choice of mesh parameters which satisfy FDM's essential conditions, and closed forms for the produced error is highly recommended and is deemed worthwhile.

\section{References}

[1] R. G. Ghanem and P. D. Spanos, Stochastic Finite Elements: A Spectral Approach, Springer, New York, NY, USA, 1991.

[2] O. H. Galal, W. El-Tahan, M. A. El-Tawil, and A. A. Mahmoud, "Spectral SFEM analysis of structures with stochastic parameters under stochastic excitation," Journal of Structural Engineering and Mechanics, vol. 28, no. 3, pp. 281-294, 2008.

[3] M. M. Kaminski, "Stochastic perturbation approach to engineering structures vibrations by the finite difference method," Journal of Sound and Vibration, vol. 251, pp. 651-670, 2002.

[4] M. Kamiński, "A generalized version of the perturbation-based stochastic finite difference method for elastic beams," Journal of Theoretical and Applied Mechanics, vol. 47, no. 4, pp. 957-975, 2009.

[5] J. Ma, J. Shen, and Y. Zhao, "On numerical approximations of forward-backward stochastic differential equations," SIAM Journal on Numerical Analysis, vol. 46, no. 5, pp. 2636-2661, 2008.

[6] S. A. Hovanessian and B. C. C. Chang, "Finite difference solution of differential equations with stochastic inputs," Journal of Theoretical and Applied Mechanics, vol. 47, pp. 957-975, 2009.

[7] A. R. Soheili, M. B. Niasar, and M. Arezoomandan, "Approximation of stochastic parabolic differential equations with two 
different finite difference schemes," Bulletin of the Iranian Mathematical Society, vol. 37, no. 2, pp. 61-83, 2011.

[8] B. A. Zeldin and P. D. Spanos, "On random field discretization in stochastic finite elements," Journal of Applied Mechanics, Transactions ASME, vol. 65, no. 2, pp. 320-327, 1998.

[9] N. Wiener, "The homogenous chaos," American Journal of Mathematics, vol. 60, pp. 897-963, 1938.

[10] R. Ghanem and P. D. Spanos, "Polynomial chaos in stochastic finite elements," Journal of Applied Mechanics, Transactions ASME, vol. 57, no. 1, pp. 197-202, 1990.

[11] R. H. Cameron and W. T. Martin, "The orthogonal development of non-linear functionals in series of Fourier-Hermite functionals," Annals of Mathematics, vol. 48, pp. 385-392, 1947.

[12] K. W. Morton and D. F. Mayers, Numerical Solution of Partial Differential Equations, Cambridge University Press, Cambridge, UK, 2nd edition, 2005.

[13] M. Shinozuka and T. Nomoto, "Response variability due to spatial randomness of material properties," Tech. Rep., Department of Civil Engineering, Columbia University, New York, NY, USA, 1980.

[14] L. Debnath, Nonlinear Partial Differential Equations for Scientists and Engineers, Birkhäuser, Boston, Mass, USA, 1st edition, 1997. 


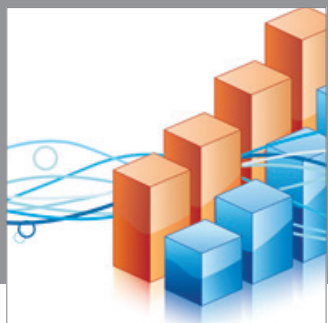

Advances in

Operations Research

mansans

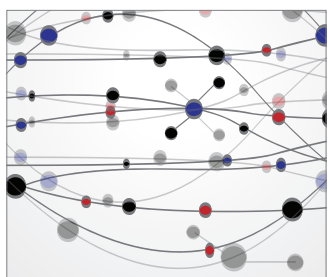

The Scientific World Journal
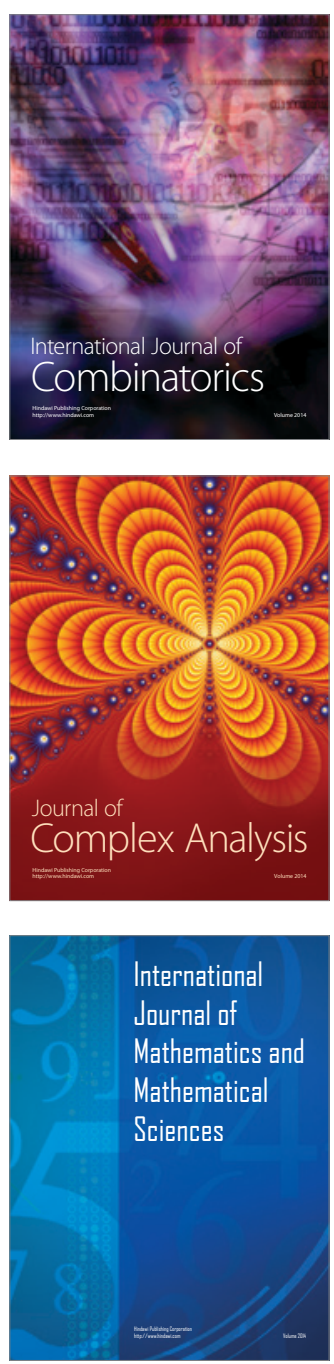
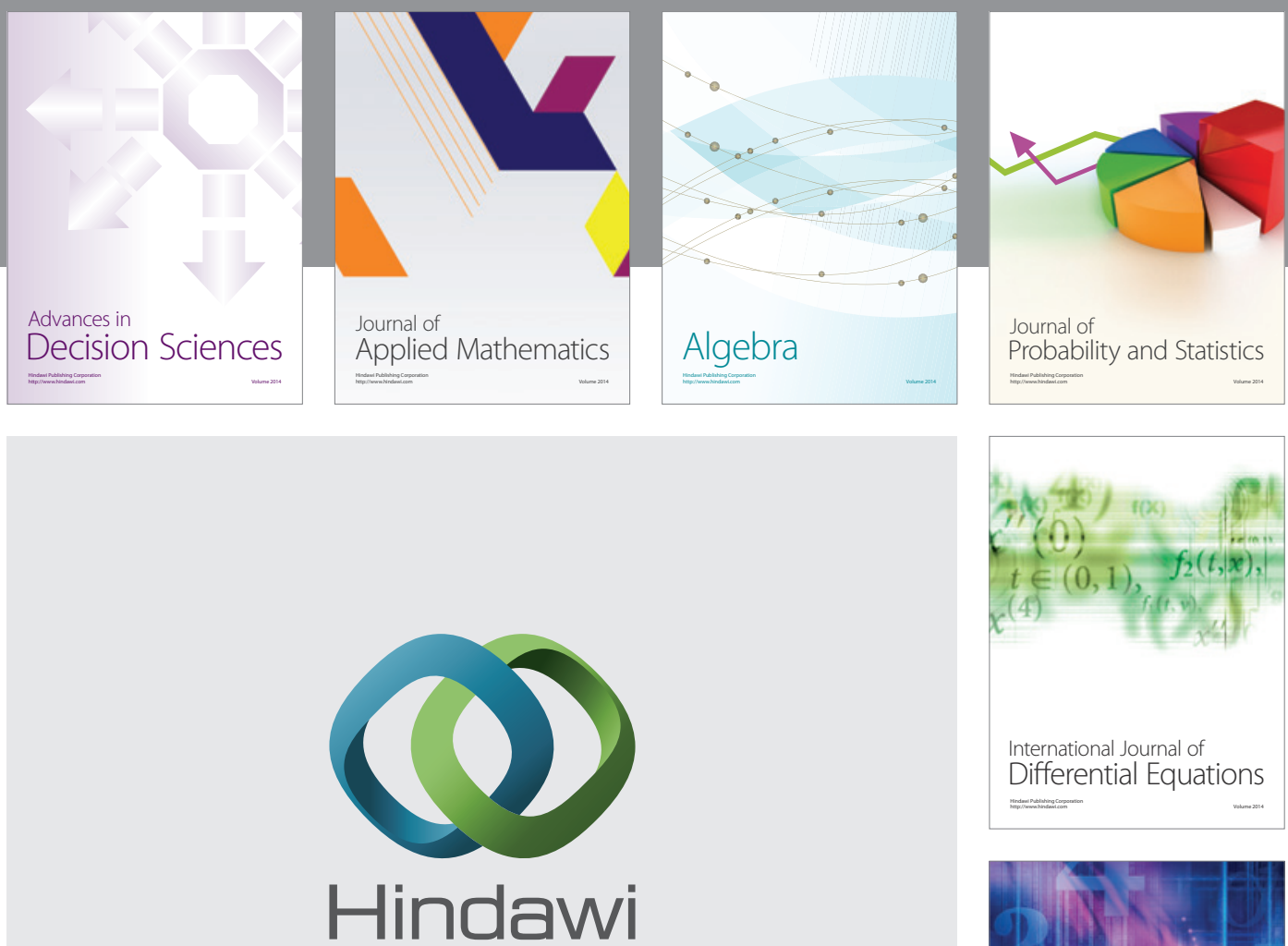

Submit your manuscripts at http://www.hindawi.com
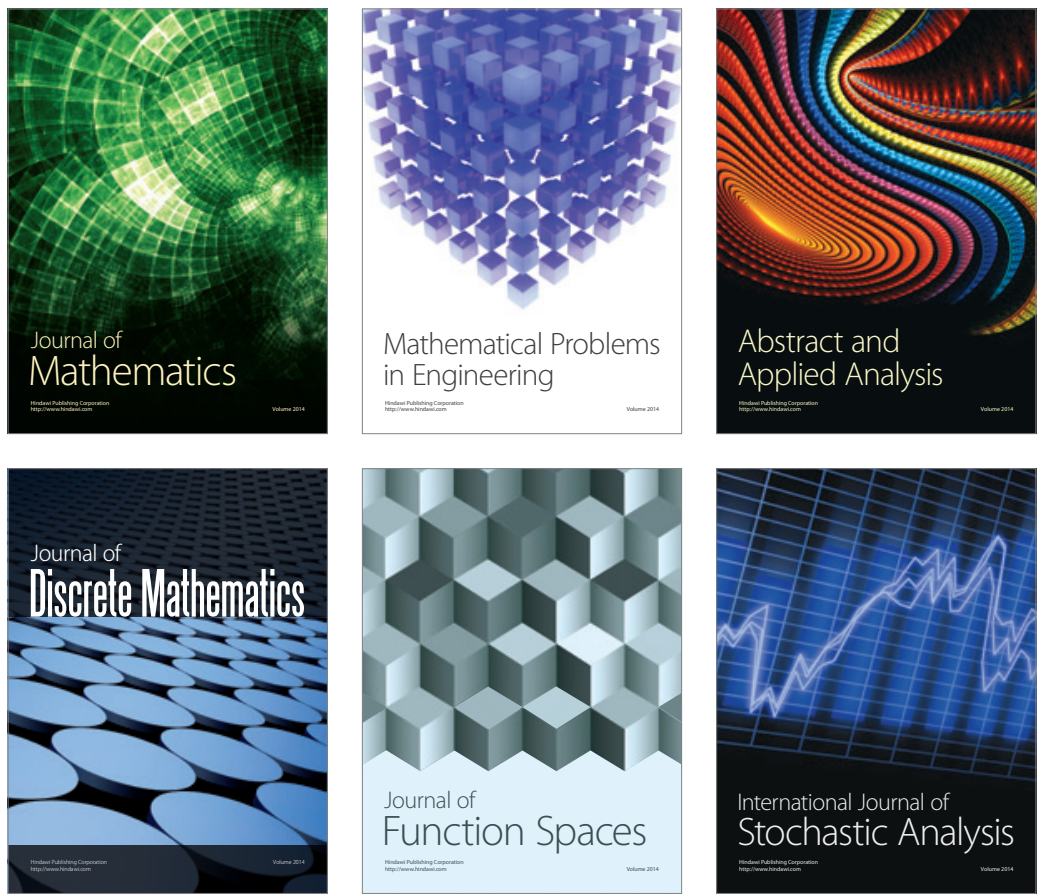

Journal of

Function Spaces

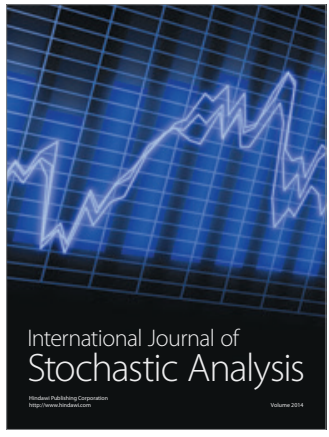

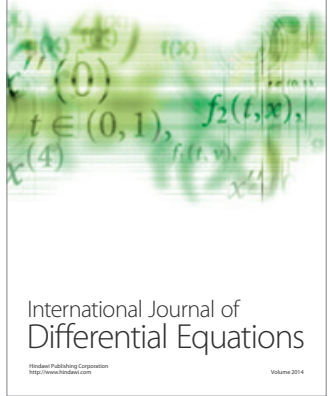
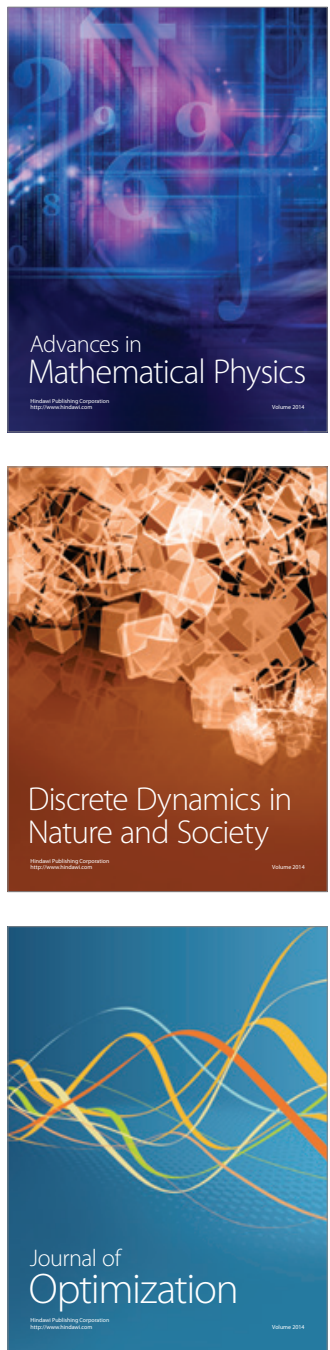